\title{
MULTI-GRID MODEL FOR CROWD'S EVACUATION IN SHIPS BASED ON CELLULAR AUTOMATA
}

\author{
Miao Chen, \\ Duanfeng Han, \\ College of Ship Building, Harbin Engineering University, \\ Harbin, China
}

\begin{abstract}
In order to enhance the authenticity and accuracy simulation of passengers' evacuation in ships, a new multi-grid model is proposed on the basis of cellular automata theory. By finer lattice the multi-grid model could enhance the continuity of passengers' track and the precision of boundary's qualification compared with traditional cellular automata model. Attraction, repulsion and friction are also quantized in the multi-grid model to present the impact of interaction force among pedestrians. Furthermore, crowd's evacuation simulated by traditional cellular automata and multi-grid model in single exit room and typical cabin environment have been taken as examples to analyze crowd's motion laws. It is found that the laws of passengers' evacuation simulated by the two models are similar, and the simulation authenticity and accuracy is enhanced by the multi-grid model.
\end{abstract}

Keywords: crowds' evacuation in ships, multi-grid model, cellular automata model, finer lattice,interaction force

\section{INTRODUCTION}

Recently well-published disasters of ships together with trends of largely increased capacity of passenger carrying ships have brought more and more attention to the issue of passengers' evacuation, being the last line of defence. It has become the investigation hotspot all around the worldwide. Mainly, there are three kinds of crowd's motion simulation construction methods. They are macroscopic models microscopic models and mesoscopic models [7].

Firstly the macroscopic model was proposed by J.J. Fruin [2]. The crowd's motion of macroscopic model is constructed by hydrodynamic theory. In macroscopic model pedestrians are taken as a whole and individual's motion detail is not considered. In the macroscopic model crowd's circulation law is simple and the simulation calculation speed is fast. It is suit for largescale pedestrians' evacuation with less individual's behavior differences. But when individual's behavior differences have a greater impact on the evacuation simulation, the defect that the macroscopic model can't simulate the behavior characteristics will be amplified and the simulation result will not match with the actual evacuation situation.

The research object of microscopic model is the single individual so the microscopic model could simulate the individual's behavior character and interactive forces between them. It is suit for simulate the evacuation in complex building environments. Social Force Model [4-6] is a microscopic model that it could simulate the individual's behavior character more realistically than any other microscopic models. It could simulate the crowds' evacuation phenomenon like fast-is-slow, self-organization, assembly, crowd congestion etc. But driving the Social Force Model needs enormous computation amount, it is not conducive to the practical engineering application.

Mesoscopic model uses a compromise approach to set up model considering the macro modeling methods and the microscopic modeling methods. It mainly refers to cellular automata models and lattice gas model. Cellular automata model $[1,3,11]$ is the general name of a kind of kinetic model with discrete process time and space. Each cell can only take a finite number of discrete states. Cellular motion models are constructed by a series of model rules. The next time step state is calculated for each cell according to the local cellular situations by the same rules and all the cells update synchronously. Cellular automata models are suit for engineering application for high calculation speed. But it often can't simulate the crowds' motion well as the movement rules are oversimplified under complex environments.

Aiming at combine the advantage of social force model and discretion model some scholars have set up new models. The floor field model and friction model can behave the self-driven force [10] and repulsive force [9] preferably. On the base of it CAFE model [12] got similar result with the social force model by quantitative analysis of repulsive force and friction between pedestrians and simulating some basic pedestrian dynamics phenomenon. Whereas those models not only could realize the dislocation distribution of pedestrians practically but also could they consider the impact of boundary qualification and exits condition sufficiently as their partitioned smallest 
grid is as big as the area taken up by one person. In order to research the characters of crowd's evacuation more carefully scholars made the grid finer that making one person taking up multi-grids method. Kirchner et al. [8] have simulated the crowd's evacuation process in corridor and single-exit room by method that one person taking up $2 \times 2$ girds. But he didn't consider the repulsion's and friction's effect. WengWenguo.etc [14] analyzed the relation between evacuation time and the number of gird moved in one time step by setting up small gird model. WeiguoSong and XuanXu et al. [13] have set up a multi-grid model that importing the reciprocity force among pedestrians and reciprocity force between pedestrians and buildings in social force model and the result of the model is close to that of social force model. But in these multi-grid model repulsion and friction was produced by pedestrians' overlapping, that didn't conform to the actual situation. In this paper a new multi-grid model is proposed with quantitative exit attraction, trajectory attraction, repulsion and friction to determine pedestrians' moving direction on the basis of cellular automata. By the model crowd's evacuation process can be simulated more accurately and realistically.

\section{MULTI GRID MODEL}

As the Fig. 1(a) shows, in single-grid model the size of single grid is the same as the area one person possessed. Each person possesses one grid and the size of each gird is $0.4 \mathrm{~m} \times 0.4 \mathrm{~m}$. But as the fig. 1(b) shows the space is further discrete by multi-gird model, the size of each gird is $0.1 \mathrm{~m} \times 0.1 \mathrm{~m}$. That is to say, each person possesses $4 \times 4$ girds and the trajectory's precision can reach $0.1 \mathrm{~m}$. At any moment, the state of each gird is empty or possessed by one person at most. As long as the neighborhood grids are empty the central pedestrian can move up and down, left and right as Fig. 2 shows.

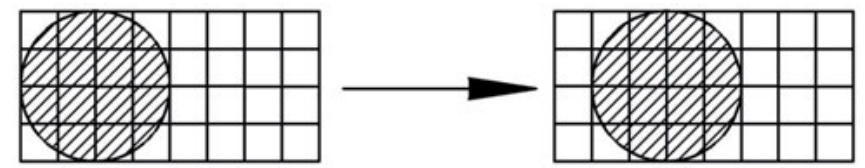

Fig. 1. Movement of pedestrian

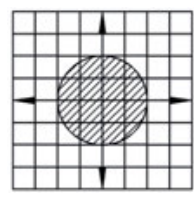

Fig. 2. direction of pedestrian's movement

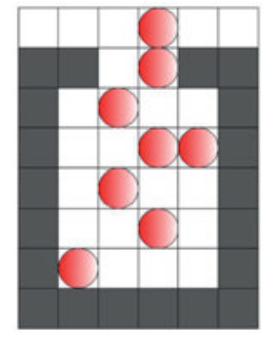

(a) single-grid model Fig. 3. Effect of exit's exclusion

\section{ADVANTAGES OF MULTI-GRID MODEL}

Compared with single-gird model, multi-gird model is improved on the continuity of pedestrian's movement, representation of reciprocity force among pedestrians and reciprocity force between pedestrians and buildings, accuracy of boundary qualification and so on. They are illustrated specifically as below.

For single-gird model each pedestrian stops or moves the length equal to a person possessed in each time step. That is coincident with the practical situation when the density of passengers is low. But the distance that a pedestrian moves could be less than the length of a person possessed when the density of passengers is high. In that situation the single-grid model can't show the fine movement of pedestrians. But for the multi-gird model, the shortest distance that a pedestrian could move is $0.1 \mathrm{~m}$. It could express the finer movement better and make the position of pedestrians vary more continuously.

For single-gird model the space between pedestrians is multiple of the length of gird. But for multi-gird model the space between pedestrians is less that the length of a person as the gird has been discrete. So the multi-gird model could realize the dislocation distribution of pedestrians.

For the multi-gird model the dislocation distribution of pedestrians has emphasized the repulsive effect between pedestrians greatly especially at the exit. As Fig. 3(a) shows it's easy for two pedestrians to pass the exit at the same time in the single-gird model. On the contrary the probability is much lower for two pedestrians to pass the exit at the same time for the effect of the dislocation distribution of pedestrians in multi-gird model. As Fig. 3(b) shows if one pedestrian possesses the middle position of the exit others can't pass through in the multi-gird model. In fact the situation is matched with the practicality. But for the single-grid model the length of exit is multiple of the length of pedestrian, the effect of exit's exclusion will not happen.

In both of the two models the size of the cabin's boundary is multiple of the size of gird. The size of gird in single-gird model is $0.4 \mathrm{~m} \times 0.4 \mathrm{~m}$ but $0.1 \mathrm{~m} \times 0.1 \mathrm{~m}$ in multi-gird model. So the accuracy of boundary qualification is detailed to $0.1 \mathrm{~m}$.

\section{CIRCULATION PRINCIPLES OF MULTI- GRID MODEL}

The movement of pedestrian is determined by probability in the multi-grid model. In each time step the center of pedestrian could stop or move to one of the adjacent 4 grids according to the state of pedestrian. As the Fig. 4 shows a preferential matrix $\mathrm{P}$ is adopted here to describe the probability value of the directions one pedestrian could move to. The value of the probability is determined by four reciprocity elements: exit attraction, trajectory attraction, repulsion and friction between pedestrians and between pedestrians and buildings. The probability of a pedestrian moving to gird $(i, j)$ can be calculated as below after considering the four elements in general. 


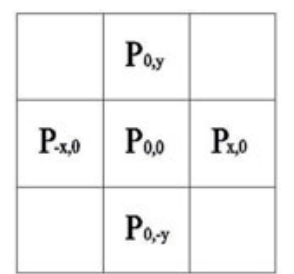

Fig. 4. Matrix $P$

$$
\begin{gathered}
p_{i j}=N \cdot \exp \left(k_{E} E_{i j}+k_{T} T_{i j}+k_{r}\left(F_{r}+F_{f}\right)\right) \cdot\left(1-n_{i j}\right) \\
N=\sum p_{i j}{ }^{-1}
\end{gathered}
$$

In formula 1 and $2, \mathrm{~N}$ the standardization coefficient can make $\sum \mathrm{p}_{\mathrm{ij}}=1 . \mathrm{n}_{\mathrm{ij}}$ represents the state of grid $(\mathrm{i}, \mathrm{j})$ at time $\mathrm{t}$. $n_{i j}=0$ shows that nobody is in grid $(i, j)$ and $n_{i j}=1$ shows that the grid $(i, j)$ is possessed by somebody or any other obstacles at the moment. As the formula 3 and 4 shows represents the character of floor and its biggest value is at the exit. $E_{i j}$ is the value of the exit attraction at the present position. E's value gradually decreases from the position of the door to a position away from the door. $\mathrm{k}_{\mathrm{E}}$ is the attraction coefficients of $\mathrm{E}_{\mathrm{ij}}$.

$$
\begin{gathered}
E_{i j}=\left|x_{i}-x_{\text {exit }}\right|+\left|y_{i}-y_{\text {exit }}\right| \\
E_{i j}=\max \left(E_{i j}\right)-E_{i j}
\end{gathered}
$$

$\mathrm{T}_{\mathrm{ij}}$ is the value of trajectory attraction and $\mathrm{T}_{\mathrm{ij}}$ represents the attraction from the trace of pedestrians. The value of $\mathrm{T}_{\mathrm{ij}}$ could be got by formula 5 . In formula $5 \varphi(\mathrm{T})$ is the $\mathrm{c}$ attenuation function and it represents the residual proportion of information seconds ago in the current time.

$$
\begin{aligned}
& D_{i j}(T)=\sum_{k=0}^{T} \varphi(T-k) D_{i j}(k) \\
& \varphi(0)=1, \quad \varphi(1)=\frac{1}{3},\left.\quad \varphi(T)\right|_{T>0}>0, \lim _{\delta T \rightarrow \infty} \varphi(T)=0
\end{aligned}
$$

Furthermore the final moving direction is also determined by repulsion's and friction's impact. $\mathrm{F}_{\mathrm{r}}$ and $\mathrm{F}_{\mathrm{f}}$ represent the repulsion and friction between pedestrians separately. $\mathrm{k}_{\mathrm{r}}$ represents the attraction coefficient of repulsion and friction.

$$
F_{r}=k_{r} \frac{1-e^{\frac{\beta}{x}}}{1+e^{\frac{\beta}{x}}}
$$

The repulsion generates when there is the trend of danger distance between pedestrians, pedestrian and walls. The pedestrian will dodge and avoid $[8,12,13,14]$. According to formula (6) each pedestrian's repulsive force can be got. In formula (6), the Sigmoid function is introduced to describe the repulsive force considering the pedestrian's avoiding behavior is a neural reflection behavior, $\beta$ is the hardness factor it relies to the pedestrians' physical endurance limitation. As Fig. 5 shows $F_{r}$ reduces when the distance between pedestrians enlarges or the value of $\beta$ decreases.

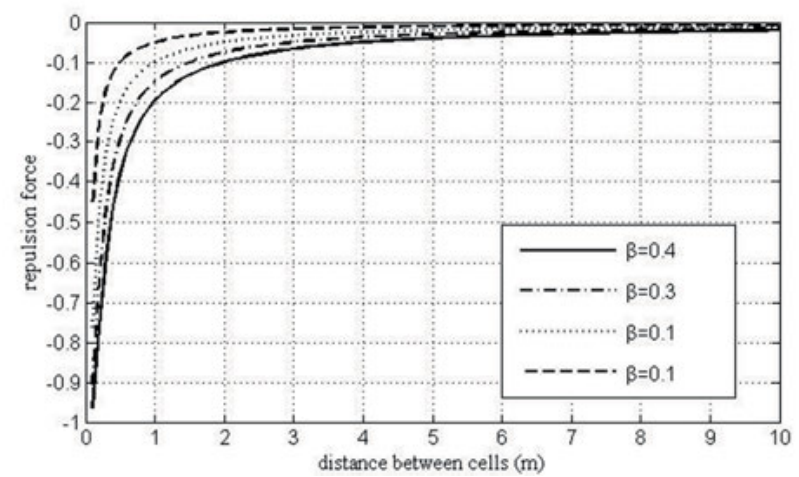

Fig.5. Impact on repulsion force for the distance between cells

According to formula 7 and 8 each pedestrian's friction can be got. The hurt triggered by the head-on collision is always bigger than the side impact triggered by friction so $\theta$ is used to link the two factors, $\theta \in[0,1]$. $\mathrm{V}$ represents the relative velocity. For two pedestrians' opposite movement $\mathrm{V}=2 \mathrm{x} v$. When one pedestrian moves and the other one stands still $\mathrm{V}=v$. For a moving pedestrian and a wall $\mathrm{V}=v . v$ is the actual speed of a pedestrian. 1 is the Line area between pedestrians and $L$ is the length of one cell as Fig. 6 shows. $\gamma$ is the repulsion ratio, $\gamma \in[0,1]$.

$$
\begin{gathered}
F_{f}=\theta F_{r} \\
\theta=\gamma \cdot\left|\frac{l}{L}\right| \cdot\left|\frac{V}{v}\right|
\end{gathered}
$$

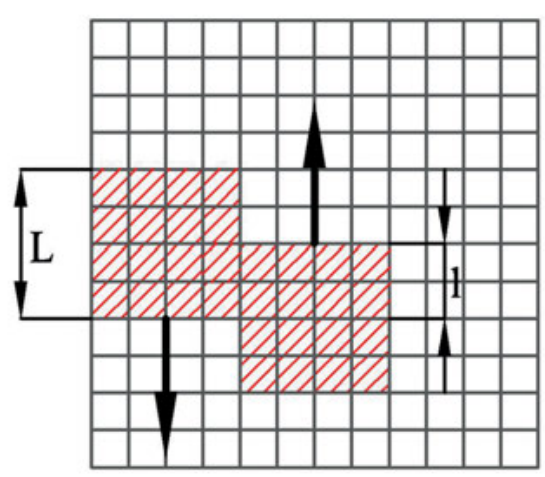

Fig.6. Friction diagram

\section{CIRCULATION PRINCIPLES OF THE MULTI-GIRD MODEL FOR MULTI-LEVEL EXITS ENVIRONMENT}

Network flow theory is adopted for simulating the arrangement of cabins in ships in traditional model. The cabins, corridors, rooms before the stairs, stairs, muster station and other construction entities are simplified to network units. As the network units are connected with doors or openings a distributed connectionist network has formed. In traditional model, each network unit has an independent self-driven field from the center of its exit. The movement is controlled by the network's self-driven field. But when the grid is discrete and the number of cabin structures is big the amount of storage 
data is great. It is too complex to simulate by traditional method since too many circulation times have decrease the efficiency in the extreme. Therefore, the self-driven fields are simplified into two: cabin field and exit field for the multilevel exits environment in this paper. The cabin field is made up of superposition of all cabins' field and the exit field is made up of superposition of all exits' field. In the process of his movement the pedestrian judges his location in the whole cabin structures firstly. If he is in a cabin he'll move to the exit of the cabin under the control of cabin floor field. Or if he is on an exit or opening he'll move to the next level cabin under the control of exit field. At last he'll reach to the final exit after such reiteration.

According to the analysis upwards, passengers' movement will be updated orderly in new principles in every time step, as the Fig. 7 shows.

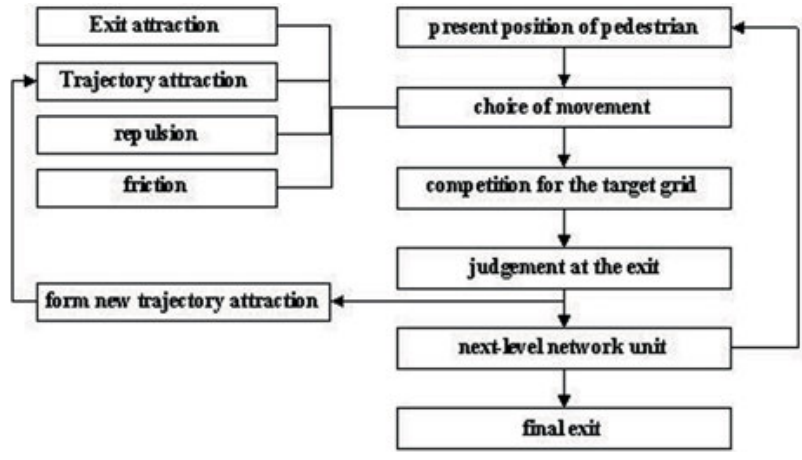

Fig. 7. Simulation principles

1) All the pedestrians choose the movement directions according to the state of adjacent girds and the principles of cabin floor field of multi-grid model.

2) According to the hypothesis one gird can only be possessed by one pedestrian in each time step. If more than one pedestrian compete for one target gird only one of them could moved to the gird and all the others have to go back the original places. In addition the chance for going to the target gird is fair to all the pedestrians.

3) When somebody reaches the exit or opening he will choose movement directions under the control of exit floor field.

4) New dynamic floor field will be formed when new trajectory is formed by pedestrians in new positions.

5) If some pedestrian moves to the next level network he will move by the principles from (1) (4) again.

6) If somebody reaches the final exit the point on behalf of the person will be removed standing for getting away from the evacuation area.
SIMULATION AND ANALYSIS

\section{EVACUATION ANALYSIS FOR SINGLE EXIT ROOM}

Tab. 1. Parameters in model

\begin{tabular}{|l|c|c|}
\hline coefficients & value & explanation \\
\hline$k_{S}$ & 1.0 & attraction coefficient of static floor field \\
\hline$k_{D}$ & 1.0 & attraction coefficient of dynamic floor field \\
\hline$k_{r}$ & 1.0 & attraction coefficient of repulsion and friction \\
\hline$\varphi$ & 0.3 & attenuation coefficient \\
\hline$\beta$ & 0.4 & hardness coefficient \\
\hline$\gamma$ & 0.3 & friction coefficient \\
\hline
\end{tabular}

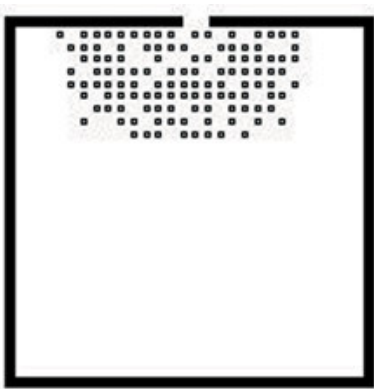

(a) traditional model

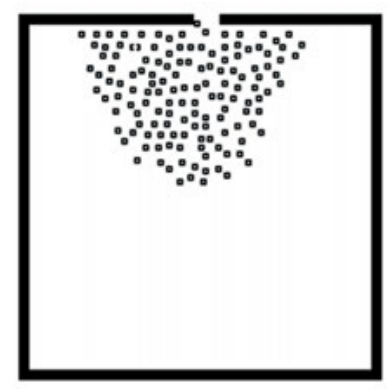

(b) multi-grid model
Fig. 8. Comparison of two models'queue in the single exit room

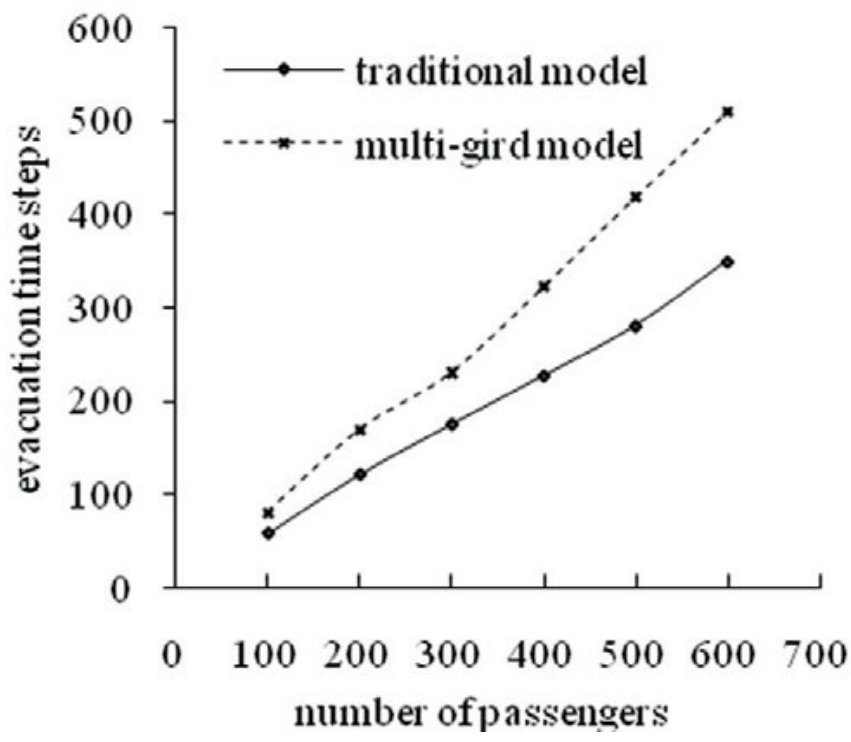

Fig. 9. Comparison of evacuation time between traditonal cellular automata model and multi-grid model

The value of coefficients is lay out above as the Tab. 1 shows. Compared with traditional cellular automata model the distance moved in one time step by multi-grid model doesn't match with the defined length of pedestrian, dislocation appears among pedestrians and the exit can't be made good use of as the Fig. 8 shows. So it needs more time to finish evacuation by multi-grid model. Fig. 9 shows the situation of 
evacuation of traditional cellular model and multi-grid model when pedestrian's velocity is $1 \mathrm{~m} / \mathrm{s}$. It is found that the bigger the density of passengers is the longer the evacuation time needs. It is caused of passengers' disorderly arrangement and low utilization ratio of exit.
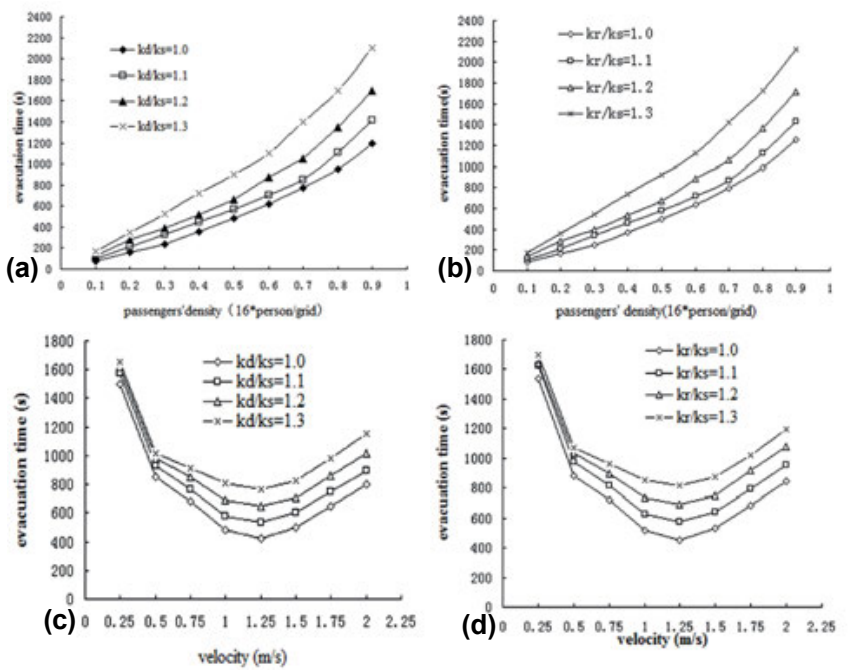

Fig. 10. The impact on evacuation time of interaction force

The value of $\mathrm{k}_{\mathrm{r}} / \mathrm{k}_{\mathrm{s}}$ and $\mathrm{k}_{\mathrm{D}} / \mathrm{k}_{\mathrm{s}}$ have an important impact on evacuation time to multi-grid model. As Fig. 10 shows, as the density of passengers increases the evacuation time increases more quickly when the value of $\mathrm{k}_{\mathrm{r}} / \mathrm{k}_{\mathrm{s}}$ and $\mathrm{k}_{\mathrm{D}} / \mathrm{k}_{\mathrm{s}}$ increase. But as the maximum velocity, $\mathrm{k}_{\mathrm{r}} / \mathrm{k}_{\mathrm{s}}$ and $\mathrm{k}_{\mathrm{D}} / \mathrm{k}_{\mathrm{s}}$ increases the evacuation time demonstrate the trend of rising before inhibition. At the same time $\mathrm{k}_{\mathrm{r}} / \mathrm{k}_{\mathrm{s}}$ have greater impact on evacuation time for the effect of friction.

\section{EVACUATION ANALYSIS FOR TYPICAL CABIN ARRANGEMENT ENVIRONMENT}

Considering the fire prevention the cabin environment of large vessels is usually separated into several main vertical zones. Each main vertical zone is closed and so independent aisles are formed. At the same time a typical cabin arrangement environment is formed on each deck of some main vertical zone according to the symmetry of cabins. Fig. 11 shows a typical cabin arrangement environment. It is made up of 42 cabins, 2 corridors, 1 room before stairs and two stairs. The area of it is $44 \mathrm{~m} \times 20 \mathrm{~m}$, the size of each cabin is $4 \mathrm{~m} \times 4 \mathrm{~m}$, the width of corridor is $1.6 \mathrm{~m}$, the width of each cabin's door is $1.2 \mathrm{~m}$ and the width of stairs is $2 \mathrm{~m}$. It is supposed that the movement of ship doesn't have an effect on the velocity of pedestrians, cabins are not on fire and there is no other passenger impact the movements in the typical cabin arrangement environment after the passengers move to the stairs.

All the passengers are distributed randomly in the 42 cabins before evacuation. They move to the stairs after receiving the evacuation information by the velocity of $1 \mathrm{~m} / \mathrm{s}$. Fig. 12 shows the evacuation process of traditional cellular automata model and multi-gird model when the density of pedestrians is $20 \%$ girds occupied. It is found that the arrangement of passengers in multi-grid model is much tighter than that in traditional model. The phenomenon of the passengers' arrangement in multi-grid model is more in line with practical situation. The main reason is that pedestrians in multi-grid model have more optional movement directions and dislocation distribution of pedestrians and better continuity movement of pedestrians can realized by discretion of grid.
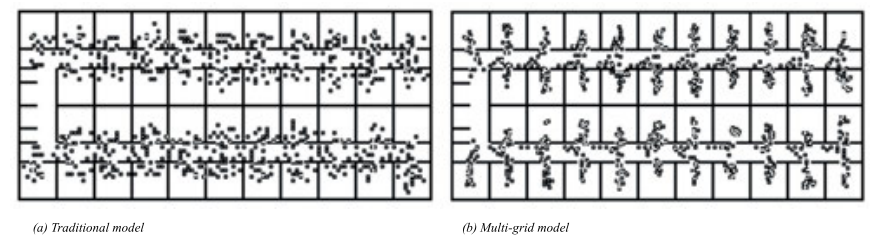

Fig.11. Evacuation of passengers

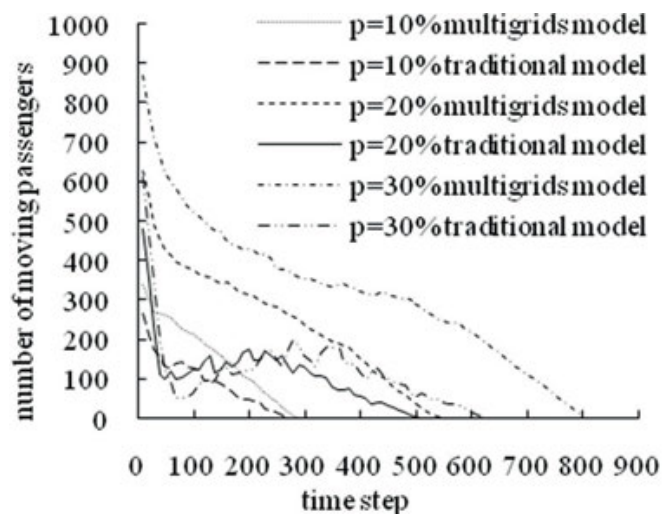

Fig. 12. Moving passengers' number for different distributing mount of passengers

Fig. 12 shows the trend of moving passengers' number varying with the evacuation time with different original density of passengers. It is found that the number of moving pedestrians decreased with the increase of evacuation time. In the process of evacuation the number of moving passenger decreases as a lot of passengers moved to the long and narrow corridors. But the crowd situation is lightened when more and more passengers finish the evacuation task. For the traditional model the reduction of the number of moving pedestrians has a repeated process. But for the multi-grid model the speed of reduction of the passengers' number is quicker for more optional movement directions, and smaller movement distance $0.1 \mathrm{~m}$. After all, the trend of the number of moving passengers' variation doesn't change.

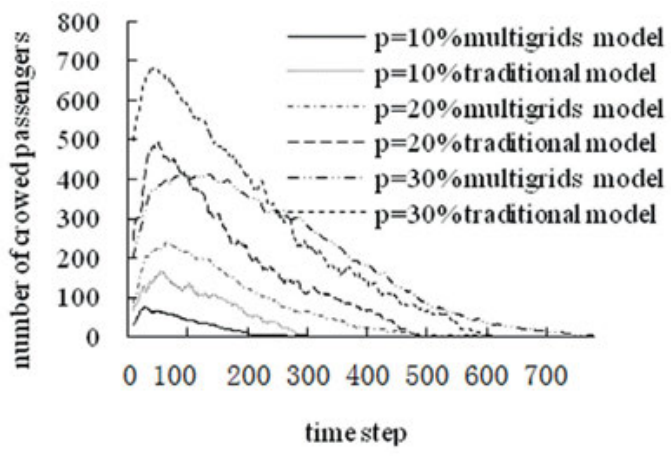

Fig. 13. Congested passengers' number for different distributing mount of passengers 


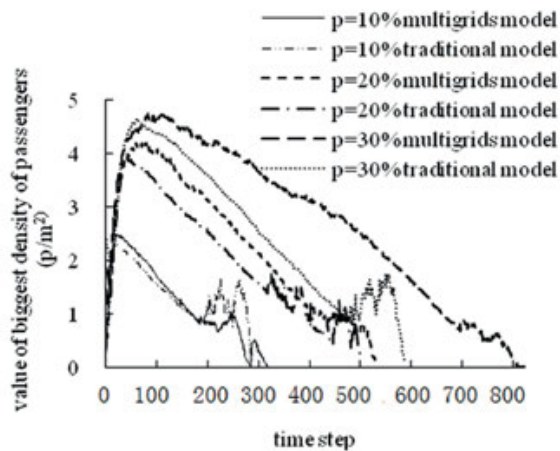

Fig. 14. Maximum density of passengers for different distributing mount of passengers

Fig.13 shows the trend of crowed pedestrians number varying with the evacuation time with different density of passengers. For the two models the number of crowed passengers increases firstly and decreased then till 0 as the increase of evacuation time. But for multi-grid model the number of crowed pedestrians is lower when the original density of passengers is the same for higher movement continuity.

Fig. 14 shows the trend of the maximum density varying with evacuation time with different original density of passengers. In the beginning of evacuation process the maximum density appears in the cabins. As more and more passengers moved to the corridors the maximum passengers density transformed to the corridors. And then the passengers' density decreased in the corridors for more and more passengers finish the evacuation task. Till to a moment that the passengers' density in the corridors are the same to that in the rooms before stairs. The maximum will appear in corridors and the rooms before stairs alternately until all the passengers leave the final exits. For the two models their trend of the maximum density variation is close. But the value of the maximum density of multi-grid model is higher for lower utilization ratio of exits caused of dislocation distribution of passengers.

\section{CONCLUSION}

Multi-grid model can simulate the evacuation situation better than traditional cellular automata model after comparison. It could reproduce the dislocation distribution of passengers, enhance the accuracy of boundary qualification and the continuity of movement trajectory and simulate evacuation process more accurately by quantizing the interaction force between passengers. According to the evacuation simulation experiment tests it is proved that the two models have similar movement laws but the multi-grid model can finish the simulation more truly and accurately.

\section{ACKNOWLEDGMENT}

This work is supported by the Ph.D. Programs Foundation of Ministry of Education of China (Grant No.20102304110018), and supported by the Foundation Research Funds for the Central Universities (Grant No.HEUCF140107).

\section{REFERENCES}

1. Burstedde C, Klauek K, Schadschneider A and Zittartz J, Simulation of Pedestrian dynamics using a twodimensional cellular automaton, Physica A, Vol. 295, pp. 507-525, 2001.

2. Fruin J J, Pedestrian Planning and Design, Metropolitan Association of Urban Designers and Environmental Planners, New York, USA, 1971.

3. Hao Yue, Hongzhi Guan, Juan Zhang and Chunfu Shao, Study on bi-direction pedestrian flow using cellular automata simulation, Physica A, Vol. 389, pp. 527-539, 2010.

4. Helbing, D., Farkas, I., and Vicsek, T, Simulating dynamical features of escape panic, Nature, Vol.407, no. 6803, pp. 487-490, 2000.

5. Helbing D, Keltseh J, Molnar P, Modelling the evolution of human trail systems, Nature, Vol.388, no. 6637, pp. 47-50. 1997.

6. Helbing D, Molnar P, Social force model for Pedestrian dynamics, Physical Review E, Vol. 51, no. 5, 4282-4286, 1995.

7. Jinyi. G, Shuang L, Shaokuan C. Review of Pedestrian Movement Simulation Studies, Jounal of System Simulation, Vol.20, no. 9, pp. 2237-2241, 2008.

8. Kirchner A, Klupfel H, Nishinari K, Schadschneider A and Schreckenberg $M$, Discretization effects and the influence of walking speed in cellular automata models for pedestrian dynamics,

9. Kirchner A, Nishinari $\mathrm{K}$ and Schadschneider A, Friction effects and clogging in a cellular automaton model for pedestrian dynamics, Physical Review E, Vol.67, no. 056122, 2003.

10. Kirchner A, Schadschneider A. Simulation of evacuation processes using a bionics-inspired cellular automaton model for pedestrian dynamics, Physica A, Vol. 312, pp.260-76, 2002.

11. Masakuni Muramatsu, Takashi Nagatani, Jamming transition of pedestrian traffic at a crossing with open boundaries, Physica A, Vol.286, pp.377-390, 2000.

12. Song WG, Yu YF, Wang BH and Fan WC, Evacuation behaviors at exit in CA model with force essentials: a comparison with social force model, Physica A, Vol. 371, pp. 658-66, 2006.

13. Journal of Statistical Mechanics-theory and Experiment J STAT MECH-THEORY EXP, vol. 10, no. 10, 2004. 
14. Weiguo Song, Xuan Xu, Bing-Hong Wang and Shunjiang $\mathrm{Ni}$, Simulation of evacuation processes using a multigrid model for pedestrian dynamics, Physica A, Vol. 363, pp.492-500, 2006.

15. W.G. Weng, L.L. Pan, S.F. Shen and H.Y. Yuan, Smallgrid analysis of discrete model for evacuation from a hall, Physica A, Vol.374, pp.821-826, 2007

\section{CONTACT WITH AUTHOR}

Miao Chen e-mail: chenmiao@hrbeu.edu.cn Mobile: 0086-18646010200

Fax: 0086-0451-82518443

Duanfeng Han e-mail: handuanfeng@hrbeu.edu.cn

College of Ship Building Harbin Engineering University Harbin, Heilongjiang Province China 